\title{
Diversity of intestinal Escherichia coli populations in Nicaraguan children with and without diarrhoea
}

\begin{abstract}
Correspondence
Inger Kühn

inger.kuhn@ki.se
\end{abstract}

Received 12 May 2009

Accepted 6 August 2009

Daniel Reyes, ${ }^{1,2}$ Samuel Vilchez, ${ }^{1,3}$ Margarita Paniagua, ${ }^{1,2}$ Patricia Colque, ${ }^{2}$ Andrej Weintraub, ${ }^{3}$ Roland Möllby ${ }^{2}$ and Inger Kühn ${ }^{2}$

\author{
${ }^{1}$ Department of Microbiology, Faculty of Medical Sciences, National Autonomous University of \\ Nicaragua (UNAN), León, Nicaragua \\ ${ }^{2}$ Department of Microbiology, Tumor and Cell Biology (MTC), Karolinska Institutet, Solna, S-171 77 \\ Stockholm, Sweden \\ ${ }^{3}$ Department of Laboratory Medicine, Division of Clinical Microbiology, Karolinska Institutet, \\ Karolinska University Hospital, Huddinge, S-141 86 Stockholm, Sweden
}

\begin{abstract}
Escherichia coli remains an important aetiological agent of infantile diarrhoea in Nicaragua. However, little is known about whether there is a high prevalence of endemic strains or whether infection is due to the epidemic spread of virulent clones. This study was undertaken to determine the diversity and distribution of clonal groups in a population of intestinal $E$. coli isolated from the faeces of children from León, Nicaragua, with $(n=381)$ and without $(n=145)$ diarrhoea, between March 2005 and September 2006. All samples had been screened previously for the presence of diarrhoeagenic E. coli (DEC) markers by multiplex PCR. From each sample, 8 E. coli colonies (where available) were analysed by biochemical fingerprinting (PhP-RE system), yielding a total of 4009 tested isolates. On average, three different biochemical phenotypes (BPTs) were found among the eight colonies analysed from each sample. The total diversity, measured as Simpson's diversity index (Di), was 0.97 among all 4009 isolates studied. Cluster analysis of data from all 4009 isolates revealed 24 common BPTs (identified in at least $1 \%$ of the isolates) and 234 less common BPTs. Similar Di values were obtained among isolates from infants with and without diarrhoea, indicating that no widespread outbreak of DEC had occurred. Moreover, among samples that were positive for the DEC types enteroaggregative E. coli, enteropathogenic E. coli and enterotoxigenic E. coli (ETEC) carrying the eltB gene, the diversities were almost as high as among non-DEC samples, whereas samples positive for ETEC carrying est $A$, enteroinvasive $E$. coli and enterohaemorrhagic $E$. coli showed lower diversities, indicating the prevalence of virulent clonal groups among these samples. The PhenePlate patterns of the 24 common BPTs identified here were compared with those obtained from $E$. coli isolated in a cohort infant study performed in 1991-1992 in the same area. Only $4 \%$ of the isolates from the 1990 s were similar to any of the common BPTs found in the present study.
\end{abstract}

\section{INTRODUCTION}

Escherichia coli is a member of the family Enterobacteriaceae encountered as a normal inhabitant of the human gut microflora. The species E. coli comprises a versatile and extremely diverse group of organisms and, in principle, each individual may harbour their own composition of strains in their gut flora. However, some strains have acquired genes that convert them to pathogens (Kuhnert et

Abbreviations: BPT, biochemical phenotype; DEC, diarrhoeagenic Escherichia coli; Di, diversity index; EAEC, enteroaggregative Escherichia coli; EHEC, enterohaemorrhagic Escherichia coli; EIEC, enteroinvasive Escherichia coli; EPEC, enteropathogenic Escherichia coli; ETEC, enterotoxigenic Escherichia coli; PhP, PhenePlate; UNAN, National Autonomous University of Nicaragua. al., 2000; Nataro \& Kaper, 1998), which may cause intestinal and extra-intestinal infection of humans and other mammals (Kaper et al., 2004; Nataro \& Kaper, 1998; Shpigel et al., 2008). Such strains are referred to as pathogenic clones and, if spread among individuals, they may cause outbreaks of disease.

In Nicaragua, previous studies on diarrhoeal illness have pinpointed diarrhoeagenic E. coli (DEC), mainly belonging to the enterotoxigenic E. coli (ETEC) and enteropathogenic E. coli (EPEC) pathotypes, as the most common bacterial causes of diarrhoea in infants. However, DECs have also been isolated frequently from healthy controls (Mayatepek et al., 1993; Paniagua et al., 1997; Vilchez et al., 2009). In order to elucidate the role of various diarrhoeal pathogens, 
including E. coli, among infants in León, Nicaragua, faecal samples from 381 children with and 145 children without diarrhoea were collected from March 2005 to September 2006, and analysed for the presence of pathogens.

In this study, we analysed the population structure of E. coli by applying a typing system [biochemical fingerprinting using the PhenePlate (PhP) system] to eight E. coli isolates from each faecal sample. In particular, we wanted to determine whether any specific clonal groups of $E$. coli dominated in samples from infants with diarrhoea. We also investigated the diversity of the faecal E. coli flora in individual infants.

\section{METHODS}

Study population and clinical assessment. This study was conducted between March 2005 and September 2006 in León, the second largest city in Nicaragua. Five health-care centres, including the emergency paediatric ward of the main hospital, were involved in this study. All enrolled participants were children with $(n=381)$ or without $(n=145)$ diarrhoea, aged $\leqslant 60$ months. A clinical examination was performed on those children who were suffering from acute diarrhoea, following the World Health Organization strategy recommendations for diarrhoea management (WHO, 2000) and adopted by the Nicaraguan health system (MINSA).

Clinical and epidemiological data were obtained from each child and registered through questionnaires. A diarrhoea case was defined as the occurrence of three or more loose, liquid or watery stools, or at least one bloody, loose stool, in the preceding $24 \mathrm{~h}$ period (WHO, 2000). Control subjects were healthy children without a history of diarrhoea for at least 1 month who were attending a health-care programme at health centres in the community. Ethics clearance for this study was approved by the Medical Bioethics Committee of the Faculty of Medical Sciences at the National Autonomous University of Nicaragua (UNAN), León, Nicaragua (registration no. 62).

Stool samples and primary microbiological procedures. Specimens were collected in plastic containers without preservatives and transported at $4{ }^{\circ} \mathrm{C}$ to the Department of Microbiology at UNAN, León, Nicaragua, on the day of collection, where primary microbiological examinations were performed. From hospitalized children, samples were collected $\leqslant 24 \mathrm{~h}$ after admission. Stools were processed and analysed within $4 \mathrm{~h}$ of collection by standard culture and identification methods (Gillespie \& Hawkey, 2006). In brief, stools were plated on MacConkey agar following overnight incubation at $37^{\circ} \mathrm{C}$. From each plate showing suspected growth of E. coli, a full loop of E. coli-like colonies was suspended in brain heart infusion broth containing $15 \%$ (v/v) glycerol, and aliquots were frozen at $-70{ }^{\circ} \mathrm{C}$. All samples were transported to the Karolinska Institutet, Stockholm, Sweden, where further characterization was performed, including screening for DEC virulence genes in the total faecal E. coli flora by multiplex PCR (Vilchez et al., 2009) and typing of the isolates using biochemical fingerprinting.

Biochemical fingerprinting of $E$. coli isolates with the PhP-RE system. After thawing, the samples were subcultured on MacConkey agar plates with overnight incubation at $37{ }^{\circ} \mathrm{C}$. From each cultured plate, eight colonies with E. coli-like morphology (where available)

Table 1. Population characteristics and total diversities of $E$. coli subpopulations

\begin{tabular}{|c|c|c|c|c|c|c|}
\hline \multicolumn{7}{|c|}{ Children with and without diarrhoea } \\
\hline \multirow[t]{2}{*}{ Age (months) } & \multicolumn{2}{|c|}{ Diarrhoea group } & \multicolumn{2}{|c|}{ Control group } & \multicolumn{2}{|c|}{ All } \\
\hline & No. of isolates & Total $\mathrm{Di}^{*}$ & No. of isolates & Total Di* & No. of isolates & Total $\mathrm{Di}^{*}$ \\
\hline$\leqslant 1$ & 57 & 0.859 & 193 & 0.947 & 250 & 0.947 \\
\hline $2-6$ & 645 & 0.958 & 345 & 0.960 & 990 & 0.964 \\
\hline $7-12$ & 753 & 0.973 & 138 & 0.952 & 891 & 0.972 \\
\hline $13-60$ & 1445 & 0.968 & 433 & 0.957 & 1878 & 0.967 \\
\hline All & 2900 & 0.970 & 1109 & 0.966 & 4009 & 0.970 \\
\hline \multicolumn{7}{|c|}{ Children with diarrhoea } \\
\hline & \multicolumn{2}{|c|}{ Hospitalized } & \multicolumn{2}{|c|}{ Not hospitalized } & \multicolumn{2}{|c|}{ All } \\
\hline & No. of isolates & Total Di* & No. of isolates & Total Di* & No. of isolates & Total Di* \\
\hline \multicolumn{7}{|c|}{ Clinical features } \\
\hline Watery stools & 499 & 0.957 & 1641 & 0.971 & 2140 & 0.960 \\
\hline Loose stools & 127 & 0.931 & 633 & 0.969 & 760 & 0.969 \\
\hline Mucus stools & 113 & 0.890 & 372 & 0.966 & 485 & 0.966 \\
\hline \multicolumn{7}{|c|}{ Therapy received $\dagger$} \\
\hline ORS & 108 & 0.954 & 2274 & 0.972 & 2382 & 0.972 \\
\hline IV fluids & 518 & 0.937 & 0 & NA & 518 & 0.937 \\
\hline All cases & 626 & 0.957 & 2274 & 0.972 & 2900 & 0.970 \\
\hline
\end{tabular}

NA, Not applicable.

*Total Di denotes the Di values calculated from all isolates in a given population.

$\dagger$ Therapy of rehydration sought depending on the dehydration status or severity of diarrhoea: ORS, oral rehydration solution; IV fluids, intravenous rehydration. 
were selected. A total of 4009 E. coli colonies (2900 from diarrhoeal and 1109 from control children; Table 1) were typed using biochemical fingerprinting (Kühn, 1985) using PhP-RE plates of the PhP system (PhPlate - http://www.phplate.se). The reagents used in the PhP-RE plates and the method of typing of $E$. coli isolates have been described previously (Achá et al., 2004; Landgren et al., 2005). In brief, each PhP-RE plate contains 8 parallel sets of 11 dehydrated reagents (carbohydrates and amino acids), which provide a high level of discrimination among E. coli strains. Growth medium containing a $\mathrm{pH}$ indicator (bromothymol blue) was added to the PhP plates, and eight E. coli colonies per plate were randomly picked and suspended into the first wells of each row of the plate. The homogenized bacterial suspensions were then transferred to the remaining wells of the same row, containing the various dehydrated reagents. Test results were read after 16,40 and $64 \mathrm{~h}$ incubation at $37^{\circ} \mathrm{C}$ using a desktop scanner (HP Scanjet 7400c XPA) with a transparency adaptor. The images generated by the scanner were converted to numerical absorbance data by PhPWIN software (PhPlate) After the last reading, the mean values from the readings of each well were calculated to give a biochemical fingerprint consisting of 11 numerical values for each isolate tested. The biochemical fingerprints of all isolates were compared pairwise with each other and the similarity between each pair was calculated as the correlation coefficient. The similarity matrix thus obtained was subjected to cluster analysis using the UPGMA (Sneath \& Sokal, 1973). Isolates showing identical biochemical fingerprints were assigned to the same biochemical phenotype (BPT). When the PhPWIN software matched at least $1 \%$ of the 4009 analysed isolates into the same BPT, a tentative common BPT was defined. BPTs found in less than $1 \%$ of isolates were termed 'others' in this study. The diversity was calculated using Simpson's index of diversity (Hunter \& Gaston, 1988), a relative measure of the distribution of isolates into different types. A high value (maximum 1) indicates an even distribution of the isolates into many different types, whereas a low value (minimum 0 ) indicates one or few dominating types in the population. All data handling, including calculations of similarities, cluster analysis and calculations of diversities, was performed using the PhPWIN software.

Statistical analysis. A non-parametric Mann-Whitney $U$-test was used for pairwise comparisons, and a $\chi^{2}$ test and Kruskal-Wallis test were used for contingency analysis, where applicable.

\section{RESULTS AND DISCUSSION}

Epidemiological typing of E. coli bacteria is normally performed on a limited number of isolates, which are subject to molecular typing methods, often PFGE (Casarez et al., 2007; Shaheen et al., 2009). More traditional methods, such as serotyping, require access to a large set of reference sera, although many isolates remain untypable. In the present study, a typing system that is able to handle a large number of isolates was needed. The $\mathrm{PhP}$ system used in this study can easily handle studies involving several thousand isolates (Ahmed et al., 2005; Landgren et al., 2005), and the software included has been designed for analysis of this number of isolates. Moreover, this $\mathrm{PhP}$ typing system has been proven to have a high discriminatory power among $E$. coli isolates of environmental as well as human origin (Landgren et al., 2005; Vilanova et al., 2004) and to be able to measure stable characteristics of the E. coli flora (Achá et al., 2005; Katouli et al., 1990).

\section{Total diversity of the E. coli populations}

Previous studies performed on E. coli using the PhP system have generated a database containing $\mathrm{PhP}$ data for more than 30000 E. coli isolates. These studies have shown that normal populations of $E$. coli of faecal origin (i.e. populations derived from non-outbreak situations) always show diversity index (Di) values above 0.96 (Achá et al., 2004; Ahmed et al., 2007; Vilanova et al., 2004). In contrast, isolates derived from infections that may be caused by virulent clones of $E$. coli show lower diversities. As an example, a study on 2481 E. coli from lower urinary tract infections yielded a total diversity of 0.94 (Landgren et al., 2005), and in other studies on ETEC from different sources, the isolates always indicated clusters of identical strains when subjected to $\mathrm{PhP}$ typing (Kühn et al., 1985; Kühn \& Möllby, 1986).

Thus, the total Di, as calculated in the present study, is a simple shortcut to answer the question of whether we often find the same type (strain) in different samples in a given population, indicating an epidemic outbreak among the studied samples. Table 1 shows the Di values of the E. coli populations studied as total Di (diversity among all isolates of each sample type). The total Di of all 4009 E. coli isolates was 0.97 , which is similar to values that have been obtained in other studies on normal E. coli of faecal origin using PhP-RE plates (Achá et al., 2004; Vilanova et al., 2004). The Di values among isolates from various subpopulations were also calculated (Table 1), and it was found that in some cases values lower than 0.97 were obtained, indicating that different infants were colonized by the same strains more often than in a normal population. This was observed for example in diarrhoeal children of $\leqslant 1$ month (0.859) (Table 1) and in hospitalized children with mucus stools (0.890). A slightly lower diversity (0.937) was observed among isolates from children who were attending the hospital and who received intravenous rehydration due to severe diarrhoea than among those children who visited a health-care centre in the community, possibly indicating either transmission and/or colonization with virulent $E$. coli clones that were capable of causing severe dehydration among this group, or that colonization by strains of hospital origin has occurred in some infants. Moreover, isolates from infants with diarrhoea who had received treatment with the antibiotics ampicillin and amoxicillin at least 1 month before the present diarrhoea episode showed low diversities (0.89 and 0.85 , respectively; data not shown). This could be due to transmission of antibioticresistant clones among these infants as a selection pressure in favour of E. coli strains possessing genes priming for resistance.

Overall, however, isolates from diarrhoeal samples showed similar Di values compared with non-diarrhoeal samples, indicating that there was no clear dominance of certain pathogenic E. coli clones among these samples. Thus, there was probably no large outbreak of DEC clones during the study period (Table 1). 


\section{Intra-diversities of $\boldsymbol{E}$. coli flora in stool samples}

The eight colonies analysed for each sample yielded a mean of three BPTs per sample. The diversities ranged between 0 (all isolates identical) and 1.0 (all isolates belonged to different BPTs) for each sample. Fig. 1 exemplifies the varying diversities in dendrograms derived from cluster analysis of $E$. coli isolates from six faecal samples, in which the BPT distributions are shown for high, medium and low diversities. There was a clear tendency, albeit nonsignificant ( $P=0.088$, Kruskal-Wallis non-parametric test), towards a lower intra-diversity in general in samples from diarrhoeal children compared with those from controls. Lower diversity would be expected if the diarrhoea was caused by overgrowth of a pathogenic E. coli clone. However, it could also be a 'wash-out effect' caused by the loose stools that eliminate transient E. coli strains in the normal flora. Intra-diversity differed significantly between ages ( $P=0.030$; Table 2). Pair-wise comparisons yielded significant increases in intra-diversities, for example between children aged $0-6$ months versus the older groups ( $P=0.04$ and $P=0.024$, respectively), indicating a less mature flora among the younger children (Alm et al., 2002). This was especially pronounced among infants aged $\leqslant 1$ month. Probably, the lower aged infants have not yet been exposed to many different $E$. coli strains and are therefore normally colonized by fewer E. coli strains (Adlerberth et al., 1998).

The intestinal E. coli flora in humans and other mammals normally contains many different $E$. coli clones, of which a few are residential (i.e. persistent over time), whereas the majority are only occasional visitors that may be found in the gut microflora on a single occasion (Adlerberth et al., 1998; Sears et al., 1950) or on a few occasions closely spaced in time after they have been ingested via food or water (Duriez et al., 2001; Gorter et al., 1998). However, the number of E. coli types detected in the gut flora increases with the number of isolates analysed. For example, Zoric et al. (2002) analysed 144 E. coli isolates from each of 6 healthy pigs using $\mathrm{PhP}$ typing and found up to 48 different BPTs per pig (Zoric et al., 2002). Thus, it is possible that important strains may escape detection, even though many colonies per sample are analysed, and searching for DEC-positive stool samples by analysing just one or two single colonies per sample would probably result in many false negatives. For the samples in the present study, PCR for DEC was performed on the total E. coli flora (Vilchez et al., 2009), which should yield a higher rate of DEC-positive samples than if performed on single colonies.

Besides low age, the factor that seemed mainly to be correlated with a low diversity of intestinal E. coli flora in
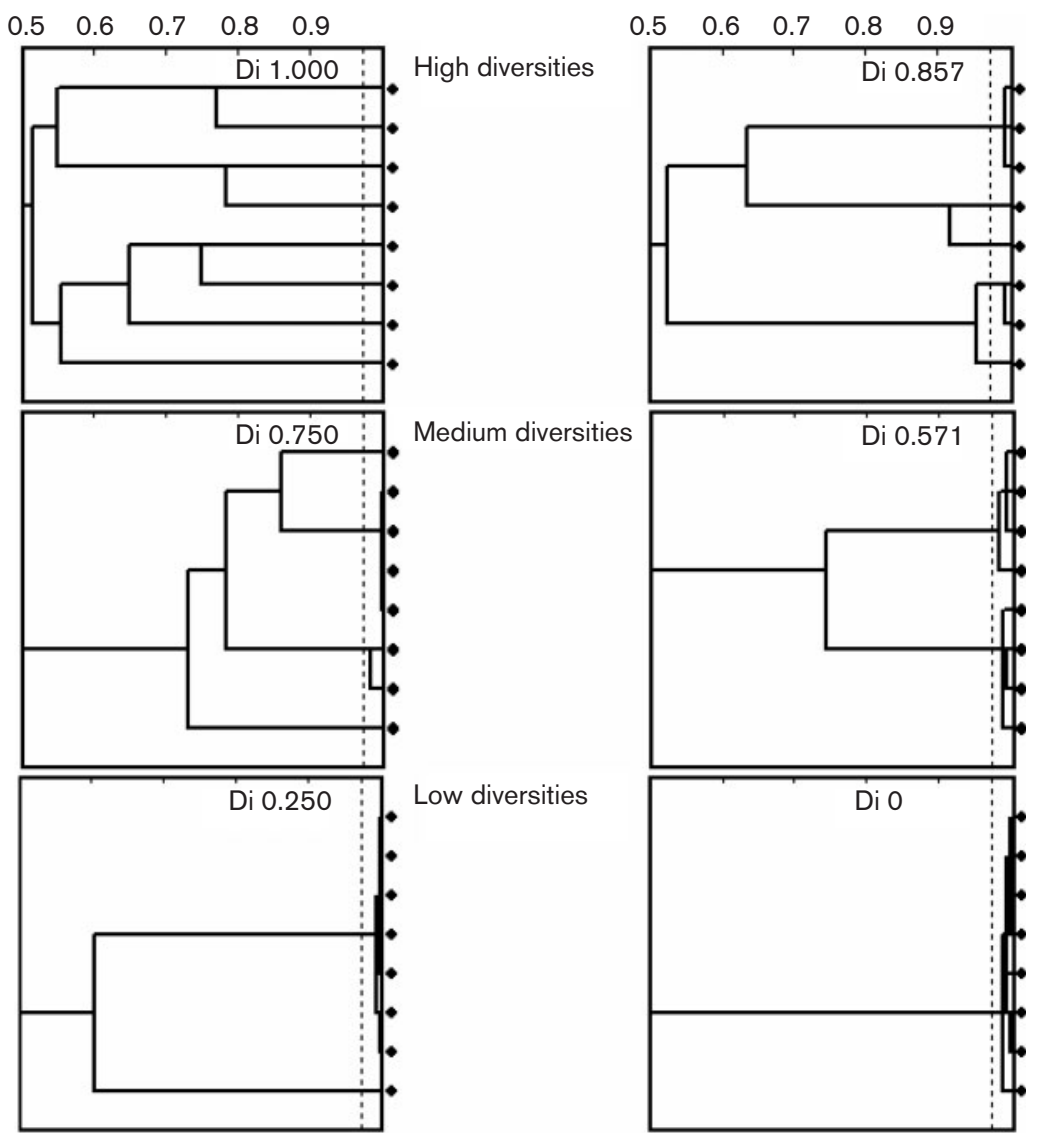

Fig. 1. Examples of dendrograms derived from UPGMA clustering of $E$. coli isolates from six faecal samples showing various degrees of intra-diversity among the eight isolates typed. Vertical dotted lines denote the pre-defined identity level. 
Table 2. Intra-diversities of $E$. coli expressed as mean Di within individual samples

Values with different superscript letters following them have significant differences between the compared age groups $(P<0.05)$.

\begin{tabular}{|c|c|c|c|c|c|c|}
\hline Age (months) & \multicolumn{2}{|c|}{ Diarrhoea group } & \multicolumn{2}{|c|}{ Control group } & \multicolumn{2}{|c|}{ All } \\
\hline$\leqslant 1$ & 8 & $0.326 \pm 0.391$ & 25 & $0.405 \pm 0.330^{c}$ & 33 & $0.386 \pm 0.341$ \\
\hline $13-60$ & 189 & $0.480 \pm 0.332^{\mathrm{b}}$ & 57 & $0.532 \pm 0.386$ & 246 & $0.492 \pm 0.345^{\mathrm{e}}$ \\
\hline All & 381 & $0.455 \pm 0.339$ & 145 & $0.508 \pm 0.355$ & 526 & $0.469 \pm 0.344$ \\
\hline \multicolumn{7}{|c|}{ Children with diarrhoea } \\
\hline & \multicolumn{2}{|c|}{ Hospitalized } & \multicolumn{2}{|c|}{ Not hospitalized } & \multicolumn{2}{|c|}{ All } \\
\hline Watery stools & 67 & $0.410 \pm 0.358$ & 214 & $0.452 \pm 0.329$ & 281 & $0.442 \pm 0.336$ \\
\hline Loose stools & 16 & $0.536 \pm 0.254$ & 84 & $0.480 \pm 0.360$ & 100 & $0.484 \pm 0.345$ \\
\hline Mucus stools & 15 & $0.503 \pm 0.334$ & 50 & $0.484 \pm 0.350$ & 65 & $0.489 \pm 0.344$ \\
\hline \multicolumn{7}{|c|}{ Therapy received $\dagger$} \\
\hline ORS & 15 & $0.547 \pm 0.371$ & 298 & $0.460 \pm 0.338$ & 313 & $0.464 \pm 0.339$ \\
\hline IV fluids & 68 & $0.410 \pm 0.334$ & 0 & $\mathrm{NA}$ & 68 & $0.410 \pm 0.334$ \\
\hline All cases & 83 & $0.434 \pm 0.343$ & 298 & $0.460 \pm 0.338$ & 381 & $0.455 \pm 0.339$ \\
\hline
\end{tabular}

${ }^{\star}$ Intra-Di denotes the mean Di value calculated from the eight isolates in individual samples.

$\dagger$ Therapy of rehydration sought depending on the dehydration status or severity of diarrhoea: ORS, oral rehydration solution; IV fluids, intravenous rehydration.

Table 3. Occurrence of virulence markers among $E$. coli flora from children with and without diarrhoea in relation to diversities of $E$. coli isolates

\begin{tabular}{|c|c|c|c|c|c|c|c|c|c|}
\hline \multirow[t]{3}{*}{ DEC type } & \multirow[t]{3}{*}{ Target gene } & \multicolumn{8}{|c|}{ Population } \\
\hline & & \multicolumn{4}{|c|}{ Diarrhoea group $(n=381)$} & \multicolumn{4}{|c|}{ Control group $(n=145)$} \\
\hline & & Samples & Isolates & Total Di $\dagger$ & Intra-Di $\neq$ & Samples & Isolates & Total Di $\dagger$ & Intra-Di申 \\
\hline EIEC & ial & $3(0.8)$ & 20 & 0.821 & 0.476 & $2(1.4)$ & 13 & 0.833 & 0.497 \\
\hline \multirow[t]{3}{*}{ ETEC } & eltB & $60(15.7)$ & 465 & 0.952 & 0.554 & $12(8.3)$ & 93 & 0.964 & 0.929 \\
\hline & est $A$ & $4(1.0)$ & 32 & 0.873 & 0.607 & $0(0.0)$ & - & - & - \\
\hline & $e l t B+e s t A$ & $14(3.7)$ & 105 & 0.912 & 0.250 & $0(0.0)$ & - & - & - \\
\hline EHEC & $v t 1, v t 2$ & $8(2.1)$ & 57 & 0.829 & 0.560 & $0(0.0)$ & - & - & - \\
\hline Non-DEC & & $176(46.2)$ & 1337 & 0.959 & 0.464 & $68(46.9)$ & 508 & 0.953 & 0.429 \\
\hline
\end{tabular}

${ }^{\star}$ The total percentage exceeds 100 , as many of the children [48 (12.6\%) with diarrhoea and $15(10.3 \%)$ controls] were positive for more than one DEC type.

$\dagger$ Total Di denotes the Di values calculated among all isolates belonging to each sample type.

¥Intra-Di denotes the mean value of the Di values calculated for the eight isolates from each individual sample. 


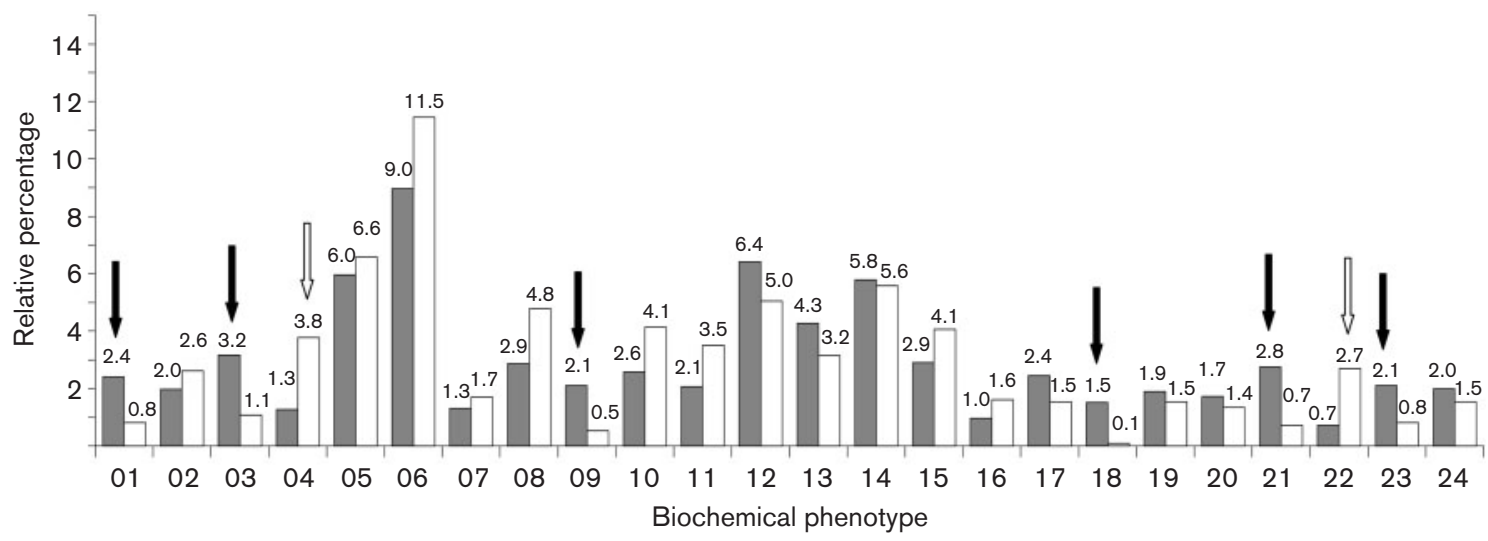

Fig. 2. Relative distribution of the most common BPTs of E. coli found among the studied population, calculated as percentages of the total number of isolates within each group (i.e. diarrhoeal and control). Black arrows indicate BPTs that were found predominantly in children with diarrhoea, and white arrows indicate BPTs that were found predominantly in control children. These BPTs were significantly different between isolates originating from diarrhoeal patients and those of controls $(P<0.01)$. Grey bars, diarrhoeal group $(n=2900)$; white bars, control group $(n=1109)$.

individual children was the presence of ETEC carrying the estA gene (Table 3). ETEC with estA were isolated only from infants with diarrhoea, and both the low total diversities among all isolates from the 18 positive children and the intra-sample diversities indicated that colonization with a limited number of pathogenic clones may have caused the diarrhoea in these children.

\section{Common BPTs}

Cluster analysis of data from all 4009 isolates revealed 24 common BPTs comprising $70 \%$ of the isolates, and 234 other BPTs found in only one or a few infants, comprising $30 \%$ of the isolates. Fig. 2 shows the relative distribution of the common BPTs among the E. coli isolates from children with and without diarrhoea. Most BPTs seemed to be distributed equally among the diarrhoeal and control children. However, some BPTs (e.g. 01, 03, 09, 18, 21 and 23) were significantly more common among children with diarrhoea than in children without diarrhoea, whereas certain BPTs (e.g. 04 and 22) were more common among the control children. The most common type found in the present study was BPT06, comprising almost 387 isolates $(9.7 \%)$. The biochemical fingerprint of this BPT could represent a clonal group of $E$. coli that is particularly common in the area of León, but it could also represent a particularly common E. coli fermentation pattern that is found in many clonal groups. Therefore, we compared the

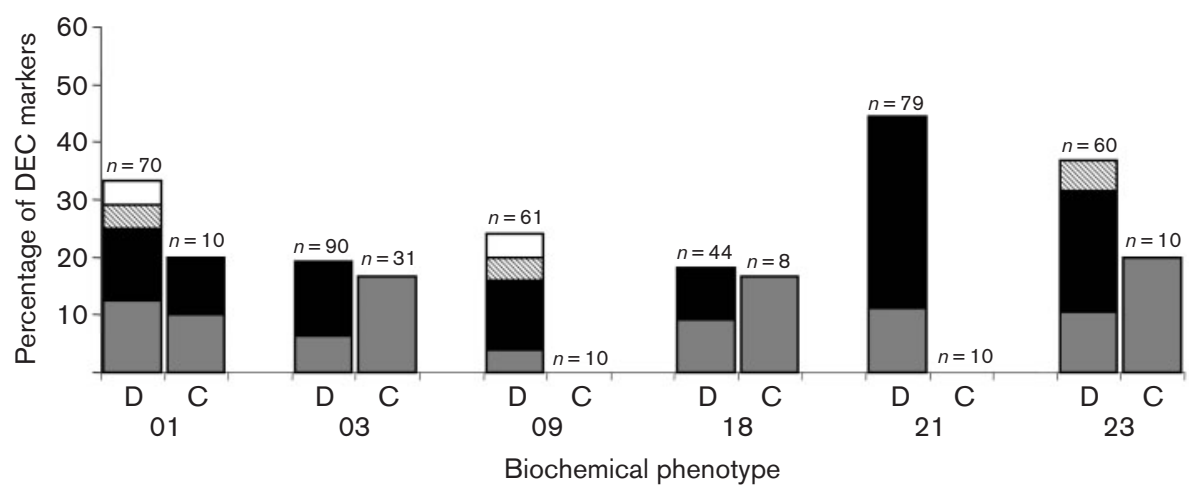

Fig. 3. BPTs of $E$. coli found predominantly in children with diarrhoea (indicated with black arrows in Fig. 2) and the proportion of samples presenting predominant DEC markers in these BPTs. The figure shows that elt $B+$ est $A$-positive samples occurred mainly in BPTs 01 and 09 (eltB, heat-labile toxin gene; est $A$, heat-stable toxin gene). Furthermore, it can be seen that not only were these BPTs found more often in diarrhoeal children (Fig. 2), but those from diarrhoeal samples were more often associated with virulence factors detected in the samples, especially types 09 and 21. D, Diarrhoeal group; C, control group. Grey bars, EPEC (eae $A$ - attaching and effacing gene); black bars, ETEC (eltB); hatched bars, ETEC (est $A$ ); white bars, ETEC $(e / t B+e s t A)$. 
biochemical fingerprint of this BPT with those obtained from 4900 isolates collected during other studies (Alm et al., 2002; unpublished data) on faecal flora of infants. Among these isolates, less than $4 \%$ matched BPT06 (data not shown), and thus the high prevalence of this BPT seems to mainly reflect an endemic origin in this studied population.

In 1991-1992, a cohort study on E. coli diarrhoea in children from León was performed (Paniagua et al., 1997) and 797 isolates from that study were also subjected to $\mathrm{PhP}$ typing. The $\mathrm{PhP}$ patterns of those isolates were compared with the 24 common BPTs identified in the present study. Only 4\% of the isolates from 1991-1992 were similar to any of the common BPTs found in 2005-2006, and only one, BPT01, was found in more than $1 \%$ of the isolates from 1991-1992 (unpublished data). This clearly illustrates that the E. coli population structure is changing continuously over time, which may be an important consideration for the development and evaluation of vaccines directed at protection against DEC infections.

\section{Total diversities of $E$. coli flora among samples presenting DEC virulence genes}

The total E. coli flora in all 526 stool samples studied had also been screened previously for DEC pathotypes by multiplex PCR (Vilchez et al., 2009). In order to study whether DEC-positive samples might contain certain clonal groups of E. coli, the diversities among the E. coli isolates in these samples were calculated separately (Table 3). Enteroaggregative E. coli (EAEC) was the most common pathotype, and the E. coli isolates from EAECpositive samples showed the same diversity as isolates from non-DEC samples. EAEC also showed a similar prevalence among diarrhoeal and control children. These findings reflect the heterogeneous nature of this E. coli type, as reported elsewhere (Nataro et al., 1995; Suzart et al., 2001). Hence, further characterization would be required to detect truly virulent EAEC strains.

Isolates from samples positive for EPEC eaeA and ETEC elt $B$ showed somewhat decreased diversities, whereas the low diversities among isolates from samples positive for ETEC est A were a clear indication that a limited number of BPTs were carriers of these virulence markers. These BPTs could represent pathogenic clonal groups that have spread among the infants (Table 3, Fig. 3). In addition, isolates from samples positive for enterohaemorrhagic E. coli (EHEC) and enteroinvasive E. coli (EIEC) showed low diversities, also indicating the presence of pathogenic clones. However, the number of samples containing these DEC types was rather small (Table 3).

In Fig. 4, an example of clustered $\mathrm{PhP}$ data is shown. The figure shows all 57 isolates from the 8 samples that were EHEC positive with the multiplex PCR screening. The dendrogram contains a cluster of 25 identical or similar isolates, derived from 6 out of 8 EHEC-positive samples, which might represent a clonal group of EHEC in this population.

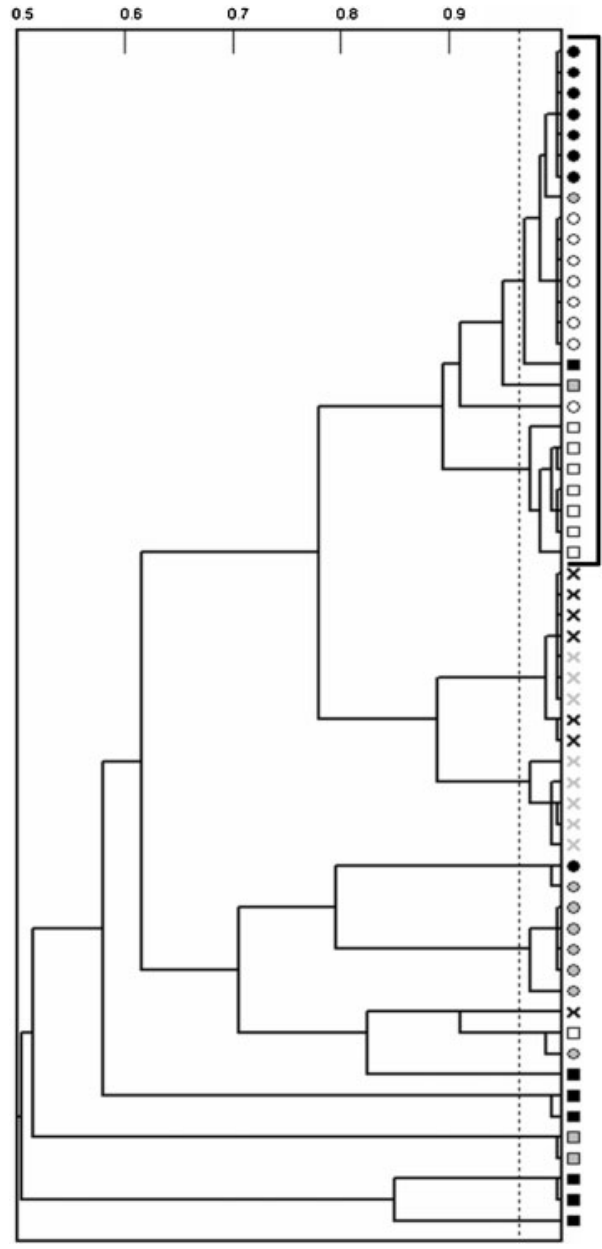

Fig. 4. Dendrogram depicting 57 E. coli isolates from 8 infant stool samples that were EHEC positive by multiplex PCR screening. The eight different symbols indicate isolates from the eight samples. The total diversity among the isolates was 0.829 . The figure shows a cluster of identical or similar isolates (vertical solid line) from six different infants. These isolates could represent a virulent clonal group.

Thus, the total diversities and distribution of BPTs found in the present study give no indication that diarrhoea in infants in León during 2005-2006 was due to any large outbreaks of pathogenic E. coli, although a large number of faecal samples contained DECs. The high prevalence of DEC-positive samples among both healthy controls and diarrhoeal infants could be due to a high endemic prevalence of many different DEC clones in this area that has resulted in a high rate of asymptomatic carriers. The fact that a significantly high proportion of infants with diarrhoea were colonized simultaneously with several DECs (Vilchez et al., 2009) supports the suggestion that the infections could have been due to ingestion of contaminated food or water (Duriez et al., 2001; Gorter et al., 1998), rather than a result of horizontal transmission between individuals. 


\section{ACKNOWLEDGEMENTS}

The authors are grateful to Patricia Blandón Roiz and Soledad Calderón for their excellent technical laboratory assistance, and to Silvia Altamirano for her valuable fieldwork activities in sample collection and transportation. We also thank the children and their parents for participating in this study. The work described in this study was supported by the Swedish Agency for Research Cooperation with Developing Countries (grant 2004-0671-75007292) and UNAN, León, Nicaragua.

\section{REFERENCES}

Achá, S. J., Kühn, I., Jonsson, P., Mbazima, G., Katouli, M. \& Möllby, R. (2004). Studies on calf diarrhoea in Mozambique: prevalence of bacterial pathogens. Acta Vet Scand 45, 27-36.

Achá, S. J., Kühn, I., Mbazima, G., Colque-Navarro, P. \& Möllby, R. (2005). Changes of viability and composition of the Escherichia coli flora in faecal samples during long time storage. J Microbiol Methods 63, 229-238.

Adlerberth, I., Jalil, F., Carlsson, B., Mellander, L., Hanson, L. A., Larsson, P., Khalil, K. \& Wold, A. E. (1998). High turnover rate of Escherichia coli strains in the intestinal flora of infants in Pakistan. Epidemiol Infect 121, 587-598.

Ahmed, W., Neller, R. \& Katouli, M. (2005). Host species-specific metabolic fingerprint database for enterococci and Escherichia coli and its application to identify sources of fecal contamination in surface waters. Appl Environ Microbiol 71, 4461-4468.

Ahmed, W., Tucker, J., Bettelheim, K. A., Neller, R. \& Katouli, M. (2007). Detection of virulence genes in Escherichia coli of an existing metabolic fingerprint database to predict the sources of pathogenic $E$. coli in surface waters. Water Res 41, 3785-3791.

Alm, J. S., Swartz, J., Björkstén, B., Engstrand, L., Engström, J., Kühn, I., Lilja, G., Möllby, R., Norin, E. \& other authors (2002). An anthroposophic lifestyle and intestinal microflora in infancy. Pediatr Allergy Immunol 13, 402-411.

Casarez, E. A., Pillai, S. D. \& Di Giovanni, G. D. (2007). Genotype diversity of Escherichia coli isolates in natural waters determined by PFGE and ERIC-PCR. Water Res 41, 3643-3648.

Duriez, P., Clermont, O., Bonacorsi, S., Bingen, E., Chaventre, A., Elion, J., Picard, B. \& Denamur, E. (2001). Commensal Escherichia col isolates are phylogenetically distributed among geographically distinct human populations. Microbiology 147, 1671-1676.

Gillespie, S. H. \& Hawkey, P. M. (2006). Principles and Practice of Clinical Bacteriology, 2nd edn. Chichester: Wiley.

Gorter, A. C., Sandiford, P., Pauw, J., Morales, P., Pérez, R. M. \& Alberts, H. (1998). Hygiene behaviour in rural Nicaragua in relation to diarrhoea. Int J Epidemiol 27, 1090-1100.

Hunter, P. R. \& Gaston, M. A. (1988). Numerical index of the discriminatory ability of typing systems: an application of Simpson's index of diversity. J Clin Microbiol 26, 2465-2466.

Kaper, J. B., Nataro, J. P. \& Mobley, H. L. (2004). Pathogenic Escherichia coli. Nat Rev Microbiol 2, 123-140.

Katouli, M., Kühn, I. \& Möllby, R. (1990). Evaluation of the stability of biochemical phenotypes of Escherichia coli upon subculturing and storage. J Gen Microbiol 136, 1681-1688.

Kühn, I. (1985). Biochemical fingerprinting of Escherichia coli: a simple method for epidemiological investigations. J Microbiol Methods 3, 159-170.
Kühn, I. \& Möllby, R. (1986). Phenotypic variations among enterotoxigenic O-groups of Escherichia coli from various human populations. Med Microbiol Immunol 175, 15-26.

Kühn, I., Franklin, A., Soderlind, O. \& Möllby, R. (1985). Phenotypic variations among enterotoxinogenic Escherichia coli from Swedish piglets with diarrhoea. Med Microbiol Immunol 174, 119-130.

Kuhnert, P., Boerlin, P. \& Frey, J. (2000). Target genes for virulence assessment of Escherichia coli isolates from water, food and the environment. FEMS Microbiol Rev 24, 107-117.

Landgren, M., Odén, H., Kühn, I., Osterlund, A. \& Kahlmeter, G. (2005). Diversity among 2481 Escherichia coli from women with community-acquired lower urinary tract infections in 17 countries. J Antimicrob Chemother 55, 928-937.

Mayatepek, E., Seebass, E., Hingst, V., Kroeger, A. \& Sonntag, H. (1993). Prevalence of enteropathogenic and enterotoxigenic Escherichia coli in children with and without diarrhoea in Esteli, Nicaragua. J Diarrhoeal Dis Res 11, 169-171.

Nataro, J. P. \& Kaper, J. B. (1998). Diarrheagenic Escherichia coli. Clin Microbiol Rev 11, 142-201.

Nataro, J. P., Deng, Y., Cookson, S., Cravioto, A., Savarino, S. J., Guers, L. D., Levine, M. M. \& Tacket, C. O. (1995). Heterogeneity of enteroaggregative Escherichia coli virulence demonstrated in volunteers. J Infect Dis 171, 465-468.

Paniagua, M., Espinoza, F., Ringman, M., Reizenstein, E., Svennerholm, A. M. \& Hallander, H. (1997). Analysis of incidence of infection with enterotoxigenic Escherichia coli in a prospective cohort study of infant diarrhea in Nicaragua. J Clin Microbiol 35, 1404-1410

Sears, H. J., Brownlee, I. \& Uchiyama, J. K. (1950). Persistence of individual strains of Escherichia coli in the intestinal tract of man. J Bacteriol 59, 293-301.

Shaheen, H. I., Abdel Messih, I. A., Klena, J. D., Mansour, A., ElWakkeel, Z., Wierzba, T. F., Sanders, J. W., Khalil, S. B., Rockabrand, D. M. \& other authors (2009). Phenotypic and genotypic analysis of enterotoxigenic Escherichia coli in samples obtained from Egyptian children presenting to referral hospitals. J Clin Microbiol 47, 189-197.

Shpigel, N. Y., Elazar, S. \& Rosenshine, I. (2008). Mammary pathogenic Escherichia coli. Curr Opin Microbiol 11, 60-65.

Sneath, P. \& Sokal, R. (1973). Numerical Taxonomy: the Principles and Practice of Numerical Classification. San Francisco, CA: W. H. Freeman.

Suzart, S., Guth, B. E., Pedroso, M. Z., Okafor, U. M. \& Gomes, T. A. (2001). Diversity of surface structures and virulence genetic markers among enteroaggregative Escherichia coli (EAEC) strains with and without the EAEC DNA probe sequence. FEMS Microbiol Lett 201, 163-168.

Vilanova, X., Manero, A., Cerdà-Cuéllar, M. \& Blanch, A. R. (2004). The composition and persistence of faecal coliforms and enterococcal populations in sewage treatment plants. J Appl Microbiol 96, 279-288.

Vilchez, S., Reyes, D., Paniagua, M., Bucardo, F., Möllby, R. \& Weintraub, A. (2009). Prevalence of diarrhoeagenic Escherichia coli in children from Leon, Nicaragua. J Med Microbiol 58, 630-637.

WHO (2000). Diarrhoea. In Handbook: IMCI - Integrated Management of Childhood Illness, pp. 18-22. WHO and Department of Child and Adolescent Health and Development (CAHD). Geneva: Department of Child and Adolescent Health and Development (CAHD), World Health Organization.

Zoric, M., Arvidsson, A., Melin, L., Kühn, I., Lindberg, J. E. \& Wallgren, P. (2002). Comparison between coliform populations at different sites of the intestinal tract of pigs. Microb Ecol Health Dis 14, 174-178. 